MATHEMATICS OF COMPUTATION

Volume 72, Number 242, Pages 677-684

S $0025-5718(02) 01435-7$

Article electronically published on May 1, 2002

\title{
OPTIMAL A PRIORI ERROR BOUNDS FOR THE RAYLEIGH-RITZ METHOD
}

\author{
GERARD L. G. SLEIJPEN, JASPER VAN DEN ESHOF, AND PAUL SMIT
}

\begin{abstract}
We derive error bounds for the Rayleigh-Ritz method for the approximation to extremal eigenpairs of a symmetric matrix. The bounds are expressed in terms of the eigenvalues of the matrix and the angle between the subspace and the eigenvector. We also present a sharp bound.
\end{abstract}

\section{INTRODUCTION}

The Rayleigh-Ritz method (or subspace projection) is a widely used technique for computing an approximation to the extreme eigenvalues and corresponding eigenvectors of a matrix $A$. It is often an integral part of modern iterative methods for computing approximations to eigenpairs of large sparse matrices. Examples of these methods for the symmetric eigenproblem include the Lanczos method [5], the Davidson method [1], and many others.

In this short paper, we derive error bounds for the Rayleigh-Ritz approximation to the eigenpair with the smallest eigenvalue of a symmetric matrix $A$. The bounds are expressed in terms of the eigenvalues of $A$ and the angle between the subspace and the eigenvector of interest. We may therefore call these bounds truly a priori. Obviously, all results can be transformed to statements about the largest eigenvalue and corresponding eigenvector by replacing $A$ with $-A$.

So, let $A$ be a symmetric matrix with eigenpairs $\left(\lambda_{i}, x_{i}\right)$ and

$$
\lambda_{1}<\lambda_{2} \leq \cdots \leq \lambda_{n-1}<\lambda_{n} .
$$

Let $V \in \mathbb{R}^{k \times n}$ be an orthogonal matrix whose columns span the $k$ dimensional subspace $\mathcal{V}$. The Rayleigh-Ritz approach gives $k$ approximate eigenpairs $\left(\theta_{i}, u_{i}\right)$, the so-called Ritz pairs, by imposing the Ritz-Galerkin condition

$$
A u_{i}-\theta_{i} u_{i} \perp \mathcal{V} \text { with } u_{i} \in \mathcal{V} \backslash\{0\},
$$

or equivalently,

$$
V^{T} A V z_{i}-\theta_{i} z_{i}=0 \text { with } u_{i} \equiv V z_{i} \neq 0 .
$$

We number the Ritz values so that

$$
\theta_{1} \leq \theta_{2} \leq \cdots \leq \theta_{k-1} \leq \theta_{k}
$$

Received by the editor October 18, 2000 and, in revised form, May 29, 2001.

2000 Mathematics Subject Classification. Primary 65F15; Secondary 65F50.

Key words and phrases. Symmetric matrices, eigenvalue problem, subspace projection, Rayleigh-Ritz, error bounds.

The research of the second author was financially supported by the Dutch Scientific Organization (NWO), under project number 613.002.035. 
We are interested in the Ritz pair $\left(\theta_{\mathcal{V}}, u_{\mathcal{V}}\right)$ for which $\sin ^{2} \angle\left(u_{\mathcal{V}}, x_{1}\right)$ is minimal over all Ritz vectors. This is the pair with the Ritz vector that makes the smallest angle with $x_{1}$ over all Ritz vectors. In the ideal case we would have that $u_{\mathcal{V}}$ is a multiple of $x_{\mathcal{V}}$, where $x_{\mathcal{V}}$ is the normalized projection of $x_{1}$ on $\mathcal{V}$. This would give $\sin ^{2} \angle\left(u_{\mathcal{V}}, x_{1}\right)=\sin ^{2} \angle\left(\mathcal{V}, x_{1}\right)$, which is optimal. Unfortunately, the approximation $u_{\mathcal{V}}$ is not a multiple of $x_{\mathcal{V}}$ in general.

The following bound is discussed in Section 2 and is a consequence of well-known bounds:

$$
\sin ^{2} \angle\left(u_{1}, x_{1}\right) \leq \sin ^{2} \angle\left(\mathcal{V}, x_{1}\right)+\frac{\lambda_{n}-\lambda_{2}}{\lambda_{2}-\lambda_{1}} \sin ^{2} \angle\left(\mathcal{V}, x_{1}\right) .
$$

This upper bound shows that $u_{1}$, corresponding to the smallest Ritz value, becomes closer to $x_{1}$, when the angle between $\mathcal{V}$ and $x_{1}$ is decreased. This and the orthogonality of the Ritz vectors guarantee that for small enough angles between $\mathcal{V}$ and $x_{1}, \theta_{\mathcal{V}}$ equals $\theta_{1}$. But although (1.1) is an elegant expression, it is not sharp. Following suggestions from [8, we show that, using only the angle $\angle\left(\mathcal{V}, x_{1}\right)$ and information about the spectrum of $A$, this bound in case $\sin ^{2} \angle\left(\mathcal{V}, x_{1}\right)<\frac{\lambda_{2}-\lambda_{1}}{\lambda_{n}-\lambda_{1}}$ can be improved to

$$
\sin ^{2} \angle\left(u_{1}, x_{1}\right) \leq \sin ^{2} \angle\left(\mathcal{V}, x_{1}\right)+\frac{\kappa}{2} \tan ^{2} \angle\left(\mathcal{V}, x_{1}\right),
$$

with $\kappa \equiv \frac{\left(\lambda_{n}-\lambda_{2}\right)^{2}}{\left(\lambda_{n}-\lambda_{1}\right)\left(\lambda_{2}-\lambda_{1}\right)}$. Bound (1.2) shows that, besides a theoretical minimum of $\sin ^{2} \angle\left(\mathcal{V}, x_{1}\right)$, we cannot lose more than an additional $\frac{\kappa}{2} \tan ^{2} \angle\left(\mathcal{V}, x_{1}\right)$. This is at most a factor $2 \frac{\lambda_{n}-\lambda_{1}}{\lambda_{n}-\lambda_{2}}$ smaller than the additional term in the bound (1.1). This factor can be large (if $\lambda_{2} \approx \lambda_{n}$ ). However, the bound (1.2) itself is at most a factor two smaller than the bound in (1.1) (if $\lambda_{2} \approx \lambda_{1}$ ). The upper bound in (1.2) is also not sharp, and we derive a less elegant but optimal upper bound in Theorem 3.2 of which (1.2) is a simple corollary. We furthermore show that under this same condition on $\sin ^{2} \angle\left(\mathcal{V}, x_{1}\right), \theta_{\mathcal{V}}$ equals $\theta_{1}$. This is the subject of Section 3

The new, sharper bounds can be used to improve a priori convergence bounds for iterative eigenvalue methods. Often, the analysis of these methods can be split into the construction of an upper bound on $\sin ^{2} \angle\left(\mathcal{V}, x_{1}\right)$ and the analysis of the error contributed by the Rayleigh-Ritz method. For example, Theorem 1 in $[\underline{6}$ gives a bound for the angle between $x_{1}$ and Krylov subspaces. Combining this with (1.1) gives precisely the bound for the first eigenvector of Kaniel 3 for the Lanczos method. In the literature, these bounds are often improved by (implicitly) constructing better bounds for $\sin ^{2} \angle\left(\mathcal{V}, x_{1}\right)$. However, in this note we focus on error bounds for the Rayleigh-Ritz method, and our results are not restricted to a specific method.

\section{SOME WELL-KNOWN UPPER BOUNDS}

Suppose that the angle $\angle\left(\mathcal{V}, x_{j}\right)$ between $\mathcal{V}$ and $x_{j}$ is small. Let $\lambda_{j}$ be the eigenvalue corresponding to $x_{j}$, where $\lambda_{j}$ is possibly in the interior of the spectrum. Then we may ask if there is a Ritz value $\theta$ close to $\lambda_{j}$. A simple application of the Bauer-Fike Theorem (Th. 4.5.1 in [5]) can answer this question affirmatively: there exists a $\theta$ such that

$$
\begin{aligned}
\left|\theta-\lambda_{j}\right| & \leq\left\|\left(V^{T} A V-\lambda_{j} I\right) V^{T} x_{\mathcal{V}}\right\|_{2}=\left\|V^{T}\left(A x_{\mathcal{V}}-\lambda_{j} x_{\mathcal{V}}\right)\right\|_{2} \\
& \leq \max _{i}\left|\lambda_{j}-\lambda_{i}\right|\left|\sin \angle\left(\mathcal{V}, x_{j}\right)\right| .
\end{aligned}
$$

See Section 4 in [2] for more discussion and analysis for general matrices. 
Unfortunately, for the Ritz vectors the situation is less attractive. A small residual, $\left(V^{T} A V-\lambda_{j} I\right) V^{T} x_{\mathcal{V}}$, is not sufficient for the existence of an eigenvector of $V^{T} A V$ that makes a small angle with $V^{T} x_{\mathcal{V}}$ if there exist two Ritz values that are close to $\lambda_{j}$. Indeed, Theorem 3 in [6] gives, for all Ritz vectors $u_{l}$

$$
\sin ^{2} \angle\left(u_{l}, x_{j}\right) \leq\left(1+\frac{\left\|\left(I-V V^{T}\right) A V V^{T}\right\|_{2}^{2}}{\min _{i \neq l}\left|\lambda_{j}-\theta_{i}\right|^{2}}\right) \sin ^{2} \angle\left(\mathcal{V}, x_{j}\right) .
$$

Since this bound is sharp (see Remark 3.4 in [4]) and since there is no guarantee that $\min _{i \neq j}\left|\lambda_{j}-\theta_{i}\right|$ is not very small, this bound for $\angle\left(u, x_{j}\right)$ can become arbitrarily large. See also the discussion in Section 5 in 2. So, this suggests that it is not possible to give meaningful error bounds for eigenvectors with eigenvalues in the interior of the spectrum using information about $\angle\left(\mathcal{V}, x_{j}\right)$ and the spectrum of $A$ only. Clearly, this might well be a problem in practical applications of RayleighRitz for interior eigenpairs. On the other hand, the bound (2.2) can be used as a good a posteriori estimate when more information about the distribution of the Ritz values is at hand.

For the extremal eigenvalues the situation is different. We know from Cauchy's Theorem (Theorem 10.1.1 in [5]) that $\left|\lambda_{1}-\theta_{2}\right| \geq\left|\lambda_{1}-\lambda_{2}\right|$, and we can construct an a priori estimate for the first eigenvector. Doing this using (2.2) and Cauchy's Theorem gives, unfortunately, a large overestimation, as we will see below.

A better approach for obtaining a true a priori bound is suggested at the end of Section 11.9 in [5]. The starting point is the well-known bound (see, for example, Theorem 11.9.2 in [5]):

$$
\sin ^{2} \angle\left(u_{1}, x_{1}\right) \leq \frac{\theta_{1}-\lambda_{1}}{\lambda_{2}-\lambda_{1}} .
$$

This bound is also sharp, which can be easily seen as follows. Take for $\mathcal{V}$ the span of $u_{1}=x_{1} \cos \alpha+x_{2} \sin \alpha$ and $u_{j}=x_{j+1}$ for $j=2, \ldots, k$. It is evident that these vectors are also the Ritz vectors. For this space $\mathcal{V}, 2.3$ becomes an equality.

In the remainder of this paper we use the notation $\epsilon \equiv \sin ^{2} \angle\left(\mathcal{V}, x_{1}\right)$. In Theorem 2.1. bounds in terms of $\epsilon$ are given for the Ritz value and Ritz vector when approximating the first eigenpair.

\section{Theorem 2.1.}

$$
\begin{gathered}
\theta_{1}-\lambda_{1} \leq\left(\lambda_{n}-\lambda_{1}\right) \epsilon \\
\sin ^{2} \angle\left(u_{1}, x_{1}\right) \leq \frac{\lambda_{n}-\lambda_{1}}{\lambda_{2}-\lambda_{1}} \epsilon=\left(1+\frac{\lambda_{n}-\lambda_{2}}{\lambda_{2}-\lambda_{1}}\right) \epsilon .
\end{gathered}
$$

Furthermore, inequality 2.4 is sharp.

Proof. The minmax property for Ritz values (Theorem 10.2.1 in [5]) gives that $\theta_{1} \leq x_{\mathcal{V}}^{T} A x_{\mathcal{V}}$, where $x_{\mathcal{V}}$ is the normalized projection of $x_{1}$ on $\mathcal{V}$. This yields

$$
\theta_{1}-\lambda_{1} \leq x_{\mathcal{V}}^{T}\left(A-\lambda_{1} I\right) x_{\mathcal{V}} \leq\left(\lambda_{n}-\lambda_{1}\right) \sin ^{2} \angle\left(x_{\mathcal{V}}, x_{1}\right)=\left(\lambda_{n}-\lambda_{1}\right) \epsilon
$$

Equality in this expression is attained by considering the space $\mathcal{V}$ spanned by the vectors $u_{1}=x_{1} \sqrt{1-\epsilon}+x_{n} \sqrt{\epsilon}$ and $u_{j}=x_{j}$ for $j=2, \ldots, k$. Note that these $u_{i}$ 's are also the Ritz vectors, and $u_{1}=x_{\mathcal{V}}$. We may conclude that (2.4) is sharp.

The second statement is a combination of (2.4) and (2.3).

Although (2.5) is a combination of the sharp bounds (2.4) and (2.3), there is no guarantee that this bound is sharp itself. Since (2.3) attains equality if $u_{1}$ has a 
component in the direction of $x_{2}$, while for (2.4) equality is attained when there is a component in the direction of $x_{n}$, it is suggested that (2.5) may not be sharp. Indeed, in the next section we improve this bound and construct a sharp bound when $\epsilon<\frac{\lambda_{2}-\lambda_{1}}{\lambda_{n}-\lambda_{1}}$. Note that $(2.5)$ is not useful when this condition on $\epsilon$ is not fulfilled.

Another question that we address is whether $\theta_{\mathcal{V}}$ equals $\theta_{1}$. This is important for the selection problem, i.e. at some point, it is necessary to select the Ritz vector that makes the smallest angle with $x_{1}$.

\section{SHARP UPPER BOUNDS}

In his $\mathrm{PhD}$ thesis [8] and in the technical report 7], Smit addressed the problem of obtaining optimal bounds for the Rayleigh-Ritz process. He derived such bounds for the case $\operatorname{dim}(\mathcal{V})=2$ and generated approximations for the $k$ dimensional case $(k>$ 2 ) by numerical experiments. On the basis of his numerical results, he conjectured that when $\epsilon<\frac{\lambda_{2}-\lambda_{1}}{\lambda_{n}-\lambda_{1}}$, the optimal bound for the $k$ dimensional case equals the optimal bound for the 2 dimensional case. In this section we prove that this is indeed correct.

For convenience we use the following notation. Let $\delta_{\mathcal{V}} \equiv \min \sin ^{2} \angle\left(u_{j}, x_{1}\right)$, where the minimum is taken over all Ritz vectors $u_{j}$ with respect to $\mathcal{V}$. Put $\epsilon \mathcal{V} \equiv$ $\sin ^{2} \angle\left(\mathcal{V}, x_{1}\right)$. For $\epsilon>0$ we define

$$
\delta_{k}(\epsilon) \equiv \max \left\{\delta_{\mathcal{V}} \mid \operatorname{dim}(\mathcal{V})=k, \epsilon_{\mathcal{V}} \leq \epsilon\right\}
$$

The following theorem is an adaptation of Theorem 4.1 in [7. We give a shorter proof, and have added the statement that $\theta_{\mathcal{V}}=\theta_{1}$ in case $\epsilon<\frac{\lambda_{2}-\lambda_{1}}{\lambda_{n}-\lambda_{1}}$.

Theorem 3.1. If $\operatorname{dim}(\mathcal{V})=2$ and $0 \leq \epsilon<\frac{\lambda_{2}-\lambda_{1}}{\lambda_{n}-\lambda_{1}}$, then $\theta_{\mathcal{V}}=\theta_{1}<\lambda_{2}$. Furthermore, with $\kappa \equiv \frac{\left(\lambda_{n}-\lambda_{2}\right)^{2}}{\left(\lambda_{n}-\lambda_{1}\right)\left(\lambda_{2}-\lambda_{1}\right)}$,

$$
\delta_{2}(\epsilon)= \begin{cases}\frac{1}{2}(1+\epsilon)-\frac{1}{2} \sqrt{(1-\epsilon)^{2}-\kappa \epsilon} & \text { if } \epsilon<\frac{\lambda_{2}-\lambda_{1}}{\lambda_{n}-\lambda_{1}}, \\ \frac{1}{2}(1+\epsilon) & \text { if } \epsilon \geq \frac{\lambda_{2}-\lambda_{1}}{\lambda_{n}-\lambda_{1}} .\end{cases}
$$

Proof. Let $0<\epsilon<1$ be given (the proof for $\epsilon=0$ and $\epsilon=1$ is obvious), and let $\mathcal{V}$ be such that $\sin ^{2} \angle\left(\mathcal{V}, x_{1}\right)=\epsilon$. We derive a sharp upper bound for the approximation to $x_{1}$ by the Ritz vectors with respect to $\mathcal{V}$. Because this bound is monotonically increasing, this gives an expression for $\delta_{k}(\epsilon)$. Notice that the Rayleigh-Ritz procedure is shift invariant, and we are allowed to work with $A-\lambda_{1} I$.

Let $\left(0, x_{1}\right),\left(\mu_{1}, w_{1}\right)$ and $\left(\mu_{2}, w_{2}\right)$ be the three Ritz pairs of the shifted matrix $A-\lambda_{1} I$ with respect to the three dimensional subspace spanned by $\mathcal{V}$ and $x_{1}$, where we have numbered $\mu_{1}$ and $\mu_{2}$ so that $\mu_{1} \leq \mu_{2}$. The vectors $w_{1}$ and $w_{2}$ are normalized. It turns out that working with $w_{1}$ and $w_{2}$ simplifies the calculations a bit.

We define for each pair $(c, s)^{T}$ on the unit circle a subspace $\mathcal{V}_{s}$ as the span of

$$
v_{s}^{(1)} \equiv x_{1} \sqrt{1-\epsilon}+c w_{1} \sqrt{\epsilon}+s w_{2} \sqrt{\epsilon} \quad \text { and } \quad v_{s}^{(2)} \equiv-s w_{1}+c w_{2} .
$$

For some pair $\left(c_{0}, s_{0}\right)^{T}$ we have that $\mathcal{V}=\mathcal{V}_{s_{0}}$.

With respect to this basis, the projected matrix $A_{s} \equiv V_{s}^{T}\left(A-\lambda_{1} I\right) V_{s}$ is given by

$$
A_{s} \equiv\left[\begin{array}{cc}
\epsilon\left(c^{2} \mu_{1}+s^{2} \mu_{2}\right) & \sqrt{\epsilon} s c\left(\mu_{2}-\mu_{1}\right) \\
\sqrt{\epsilon} s c\left(\mu_{2}-\mu_{1}\right) & c^{2} \mu_{2}+s^{2} \mu_{1}
\end{array}\right]
$$


Note that if $s=0$ or $c=0$, then the vector $x_{\mathcal{V}}$ is a Ritz vector, and we can exclude this situation from our analysis.

Let $u_{i} \equiv V_{s} z_{i}$, with $z_{i}=\left(t_{i}, 1\right)^{T}$ a scaled eigenvector of the projected matrix $A_{s}$. Then

$$
\sin ^{2} \angle\left(u_{i}, x_{1}\right)=1-\frac{t_{i}^{2}}{1+t_{i}^{2}}(1-\epsilon)=\frac{1+\epsilon t_{i}^{2}}{1+t_{i}^{2}}=\frac{1}{2}(1+\epsilon)-\frac{1}{2}(1-\epsilon) \frac{t_{i}^{2}-1}{t_{i}^{2}+1} .
$$

We are interested in the smallest possible value of $\max \left\{\left|t_{1}\right|,\left|t_{2}\right|\right\}$. It suffices to analyze the eigenvectors of

$$
A_{s}^{\prime} \equiv \frac{1}{\mu_{2} c^{2}} A_{s}=\left[\begin{array}{cc}
\epsilon\left(\mu+\tau^{2}\right) & \sqrt{\epsilon} \tau(1-\mu) \\
\sqrt{\epsilon} \tau(1-\mu) & 1+\tau^{2} \mu
\end{array}\right], \quad \text { where } \quad \mu \equiv \mu_{1} / \mu_{2} \text { and } \tau=s / c
$$

The ratio of the coordinates of the equation $A_{s}^{\prime}(t, 1)^{T}=\theta^{\prime}(t, 1)^{T}$ is given by

$$
t \epsilon\left(\mu+\tau^{2}\right)+\sqrt{\epsilon} \tau(1-\mu)=t^{2} \sqrt{\epsilon} \tau(1-\mu)+t\left(\tau^{2} \mu+1\right) .
$$

The vector $(t, 1)^{T}$ is an eigenvector of $A_{s}^{\prime}$ if and only if $t$ satisfies this equation. We investigate the possible values for $t$,

$$
\frac{1-t^{2}}{t}=g(\tau) \equiv \frac{\alpha}{\tau}+\beta \tau, \quad \text { where } \quad \alpha \equiv \frac{1-\epsilon \mu}{\sqrt{\epsilon}(1-\mu)}, \quad \beta \equiv \frac{\mu-\epsilon}{\sqrt{\epsilon}(1-\mu)} .
$$

Because $\epsilon<1$ and $\mu \leq 1$, we have $\alpha>0$. We start by giving a proof for $\tau>0$.

We first consider the case where $\beta>0$, or, equivalently, $\epsilon<\mu$. Then $g(\tau)$ takes values between $2 \sqrt{\alpha \beta}$ and $\infty$. Hence, $t$ takes values between 0 and $\sqrt{\alpha \beta+1}-\sqrt{\alpha \beta}$ and between $-\infty$ and $-(\sqrt{\alpha \beta+1}+\sqrt{\alpha \beta})$. Because $z_{1} \perp z_{2}$, we know that $t_{1}=$ $-t_{2}^{-1}$, and it easily follows that there is a $t_{i}$ in each of the two intervals. Define $t_{1}$ to be in the negative interval and note that $\left|t_{1}\right|>\left|t_{2}\right|$. The value $\left|t_{1}\right|=\sqrt{\alpha \beta+1}+\sqrt{\alpha \beta}$ is the smallest possible value for $\max \left\{\left|t_{1}\right|,\left|t_{2}\right|\right\}$; this gives

$$
\frac{t^{2}-1}{t^{2}+1}=\sqrt{\frac{\alpha \beta}{\alpha \beta+1}}=\sqrt{1-\frac{(1-\mu)^{2}}{\mu} \frac{\epsilon}{(1-\epsilon)^{2}}} .
$$

Inserting this in (3.1) gives the expression for $\delta_{k}(\epsilon)$ when $\epsilon<\mu$ and $\tau>0$.

Now we show that in case $\epsilon\left\langle\mu\right.$ and $\tau>0, \theta_{\mathcal{V}}$ equals $\theta_{1}$. Let $\left(t_{i}, 1\right)^{T}$ be an eigenvector of $A_{s}$; then the second component of the vector $A_{s}\left(t_{i}, 1\right)^{T}$ gives an expression for $\theta_{i}$ :

$$
\theta_{i}=\mu_{2} c^{2}\left(t_{i} \sqrt{\epsilon} \tau(1-\mu)+1+\tau^{2} \mu\right) .
$$

If we recall the signs of $t_{i}$, we have that $\theta_{1}<\theta_{2}$, and because $\left|t_{1}\right|>\left|t_{2}\right|$ we get that $\theta_{\mathcal{V}}=\theta_{1}$

If $\beta \leq 0$, or equivalently $\epsilon \geq \mu$, then $g(\tau)$ takes all values. Therefore, $t$ can take all values between the same bounds. Consequently, there is a $\tau$ for which $t_{1}=1$ and $t_{2}=-1$ are solutions. This corresponds to the worst possible situation. In this case we have two Ritz vectors, $u_{1}$ and $u_{2}$, that make the same angle with $x_{1}$, and $\sin ^{2} \angle\left(u_{i}, x_{1}\right)=\frac{1}{2}(1+\epsilon)$.

In case $\tau<0$ the same reasoning can be used. The proof for the expression of $\delta_{k}(\epsilon)$ is concluded by noting that $\mu=\frac{\lambda_{2}-\lambda_{1}}{\lambda_{n}-\lambda_{1}}$ is the smallest possible value for $\mu$, and this is the worst situation. 
Note that the bound $(1+\epsilon) / 2$ holds for any orthogonal basis for $\mathcal{V}$. So, in case $\epsilon \geq \frac{\lambda_{2}-\lambda_{1}}{\lambda_{n}-\lambda_{1}}$, the Ritz vectors are not guaranteed to contain a better approximation than, for example, simply the columns of the matrix $V$.

Now we are ready to give a proof for Conjecture 5.1 in [7]. This conjecture states that, in case $\epsilon<\frac{\lambda_{2}-\lambda_{1}}{\lambda_{n}-\lambda_{1}}, \delta_{k}(\epsilon)=\delta_{2}(\epsilon)$. So, the expression for $\delta_{k}(\epsilon)$ is given by the expression in Theorem 3.1 .

Theorem 3.2. If $0 \leq \epsilon<\frac{\lambda_{2}-\lambda_{1}}{\lambda_{n}-\lambda_{1}}$, then $\theta_{\mathcal{V}}=\theta_{1}<\lambda_{2}$. For all $k \in\{2, \ldots, n-1\}$ and all $\epsilon \in\left[0, \frac{\lambda_{2}-\lambda_{1}}{\lambda_{n}-\lambda_{1}}\right)$, we have

$$
\delta_{k}(\epsilon) \equiv \frac{1}{2}(1+\epsilon)-\frac{1}{2} \sqrt{(1-\epsilon)^{2}-\kappa \epsilon}, \quad \text { with } \kappa \equiv \frac{\left(\lambda_{n}-\lambda_{2}\right)^{2}}{\left(\lambda_{n}-\lambda_{1}\right)\left(\lambda_{2}-\lambda_{1}\right)} .
$$

Proof. Assume that $\epsilon_{\mathcal{V}}<\frac{\lambda_{2}-\lambda_{1}}{\lambda_{n}-\lambda_{1}}$. Then $\theta_{1}<\lambda_{2}$ (see 2.4)). Consider the space $\mathcal{V}^{\prime}$ spanned by $u_{1}$ and $x_{\mathcal{U}}$, where $x_{\mathcal{U}}$ is the normalized projection on the space $\mathcal{U} \equiv \operatorname{span}\left(u_{2}, \ldots, u_{k}\right)$. Note that $u_{1}$ and $x_{\mathcal{U}}$ are Ritz vectors with respect to this 2 dimensional space $\mathcal{V}^{\prime}$. Lemma 3.1 states that for this 2 dimensional $\mathcal{V}^{\prime}$, the angle between $u_{1}$ and $x_{1}$ is less than the angle between $x_{\mathcal{U}}$ and $x_{1}$. Since the angle between $x_{\mathcal{U}}$ and $x_{1}$ is smaller than the angle between any vector from $\mathcal{U}$ and $x_{1}$, we may conclude that $\theta_{\mathcal{V}}=\theta_{1}$.

Note that $\epsilon_{\mathcal{V}}=\epsilon_{\mathcal{V}^{\prime}}$ and $\delta_{\mathcal{V}}=\delta_{\mathcal{V}^{\prime}} \leq \delta_{2}$, which implies that $\delta_{k} \leq \delta_{2}$

We now show that $\delta_{2} \leq \delta_{k}$. Let $\operatorname{dim}(\mathcal{V})=2$, then select an orthogonal system $v_{3}, \ldots, v_{k}$ that is orthogonal to $u_{1}, u_{2}$, and $A u_{1}-\theta_{1} u_{1}$. Then $\left(\theta_{1}, u_{1}\right)$ is also a Ritz pair of the space $\mathcal{V}^{\prime}$ spanned by $u_{1}, u_{2}, v_{3}, \ldots, v_{k}$. Since $\theta_{1}<\lambda_{2}$, Cauchy's Theorem (Th. 10.1.1 in [5]) guarantees that the extension does not introduce a Ritz value in $\left[\lambda_{1}, \lambda_{2}\right)$. As argued above, $\theta_{\mathcal{V}}=\theta_{\mathcal{V}^{\prime}}=\theta_{1}$. Moreover $\epsilon_{\mathcal{V}^{\prime}} \leq \epsilon_{V}$. Apparently, $\delta_{\mathcal{V}}=\delta_{\mathcal{V}^{\prime}} \leq \delta_{k}$

We have that $\delta_{2}=\delta_{k}$, and Theorem 3.1 now gives the expression for $\delta_{k}$.

We recall that the restriction on $\epsilon$ in Theorem 3.2 in this situation does not make the bounds more restrictive than the bound (2.5) in the previous section.

We mention a few consequences of Theorem [3.2. Corollaries 3.1 and 3.2 generalize Corollaries 4.3 and 4.4, respectively, in 7]. The first corollary describes the behavior of the upper bound (3.2) for small $\epsilon$.

Corollary 3.1. For all $k \in\{2, \ldots, n-1\}$, we have

$$
\delta_{k}(\epsilon)=\epsilon\left(1+\frac{1}{4} \frac{\left(\lambda_{n}-\lambda_{2}\right)^{2}}{\left(\lambda_{n}-\lambda_{1}\right)\left(\lambda_{2}-\lambda_{1}\right)}\right)+\mathcal{O}\left(\epsilon^{2}\right) \quad \text { for } \epsilon \rightarrow 0 .
$$

Proof. Indeed,

$$
\sqrt{(1-\epsilon)^{2}-\kappa \epsilon}=1-\epsilon-\frac{1}{2} \kappa \epsilon+\mathcal{O}\left(\epsilon^{2}\right) \quad \text { for } \epsilon \rightarrow 0
$$

Inserting this in (3.2) and using the definition of $\kappa$ gives the required expression.

Inequality (2.5) is of a linear form. Using Theorem 3.2, we can improve this by at most a factor two. Corollary 3.2 gives a linear bound that equals (3.2) in $\epsilon=0$ and $\epsilon=\frac{\lambda_{2}-\lambda_{1}}{\lambda_{n}-\lambda_{1}}$. Note that $\delta_{k}(\epsilon)$ is a convex function in this interval and, hence, this is the best linear bound possible.

Corollary 3.2. For all $k \in\{2, \ldots, n-1\}$ and all $\epsilon \in\left[0, \frac{\lambda_{2}-\lambda_{1}}{\lambda_{n}-\lambda_{1}}\right)$, we have

$$
\delta_{k}(\epsilon) \leq \epsilon\left(1+\frac{1}{2} \frac{\lambda_{n}-\lambda_{2}}{\lambda_{2}-\lambda_{1}}\right) .
$$



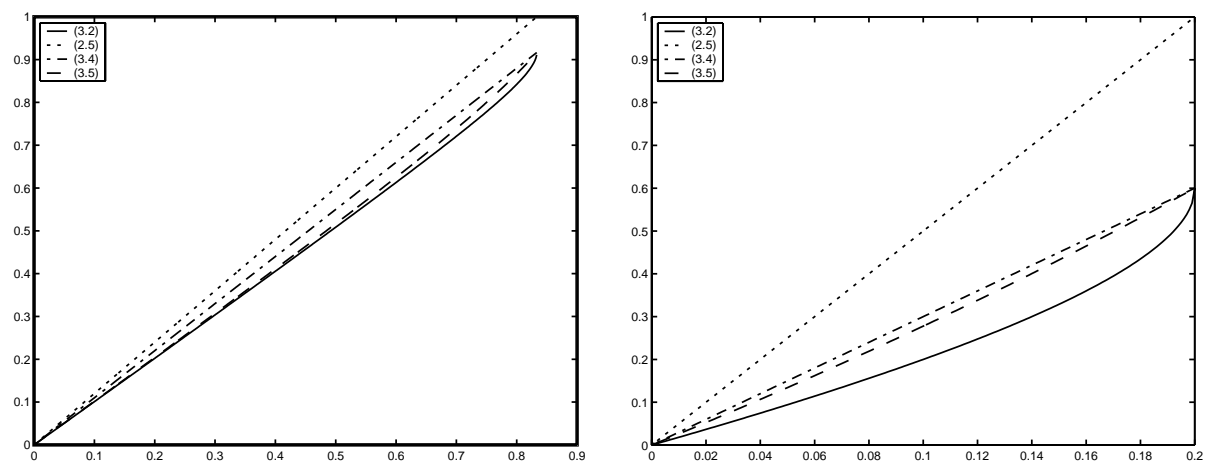

FiguRE 1. Illustration of different bounds for $\lambda_{1}=0, \lambda_{2}=1$ with $\lambda_{n}=1.2$ (left picture) and $\lambda_{n}=5$ (right picture). The numbers in the legend of the figures correspond to the number of the equation that contains the bound that is illustrated here. The bounds are along the vertical axis, and $\epsilon$ is along the horizontal axis.

The next corollary gives an upper bound for $\delta_{k}(\epsilon)$ that better approximates the optimal bound (3.2) for small $\epsilon$ and $\kappa \approx 1$.

Corollary 3.3. For all $k \in\{2, \ldots, n-1\}$ and all $\epsilon \in\left[0, \frac{\lambda_{2}-\lambda_{1}}{\lambda_{n}-\lambda_{1}}\right)$, we have

$$
\delta_{k}(\epsilon) \leq \epsilon+\frac{\kappa}{2} \frac{\epsilon}{1-\epsilon},
$$

with $\kappa \equiv \frac{\left(\lambda_{n}-\lambda_{2}\right)^{2}}{\left(\lambda_{n}-\lambda_{1}\right)\left(\lambda_{2}-\lambda_{1}\right)}$.

Proof. We rewrite the expression for $\delta_{k}(\epsilon)$ in (3.2) for $\epsilon<\frac{\lambda_{2}-\lambda_{1}}{\lambda_{n}-\lambda_{1}}$ :

$$
\delta_{k}(\epsilon)=\epsilon+\frac{1}{2}(1-\epsilon)(1-\sqrt{1-\alpha})=\epsilon+\frac{1}{2}(1-\epsilon) \frac{\alpha}{1+\sqrt{1-\alpha}} \quad \text { with } \alpha=\frac{\kappa \epsilon}{(1-\epsilon)^{2}} .
$$

Multiplying the nominator and denominator in the second term by $1-\epsilon$ and using $\kappa \epsilon<(1-\epsilon)^{2}$ gives the first inequality.

To give some feeling for the quality of the different bounds, we have illustrated in Figure 1 the known bound (2.5) and the new bounds (3.2), (3.4), and (3.5) for a matrix with $\lambda_{1}=0, \lambda_{2}=1$ and two values for $\lambda_{n}, \lambda_{n}=1.2$ and $\lambda_{n}=5$. The left picture shows that for well conditioned eigenvectors $\left(\left(\lambda_{n}-\lambda_{1}\right) /\left(\lambda_{2}-\lambda_{1}\right) \approx 1\right)$, our bounds do not improve much on the straightforward bound from the previous section. In the right picture, the ratio between spread and gap is a little larger and the improvement is more apparent. Note that the first two terms of the expansion of $\delta_{k}(\epsilon)$ in (3.3) provide a lower bound on $\delta_{k}(\epsilon)$. This shows that the bound in (2.5) can be improved at best by a factor 4 .

With respect to the problem of selection, choosing the smallest Ritz pair seems safe and guarantees correct selection asymptotically.

\section{Future Research: Harmonic Ritz vectors}

Krylov subspaces tend to contain good approximations to the extremal eigenpairs first. In this situation the Ritz vector can be deflated and our results can be applied to get statements for the next "extremal" eigenpair, and so on. However, if deflation is not possible, or not efficient, then other tools are required for eigenvalues that are more in the interior of the spectrum. 
The goal of this study was to clear the way for studying the more complicated harmonic Ritz vectors. The harmonic Ritz pairs are the Ritz pairs with respect to the search space $\mathcal{V}$ and test space $(A-\tau I) \mathcal{V}$. The idea behind this is that, if $\lambda$ is the closest eigenvalue to $\tau$ and $\lambda$ is simple, only one harmonic Ritz value can get arbitrary close to $\lambda$. The lack of guaranteed separation of the Ritz values is the reason why there is a problem with constructing true a priori bounds for Ritz vectors with eigenvalues in the interior of the spectrum. Although bounds like (2.2) cannot be used in the context of harmonic Ritz vectors, practical observations indeed suggest that there always seems to be a good harmonic Ritz vector. Understanding this by straightforwardly applying well-known techniques, as in Section 2, seems to give large overestimations. The technique described in Section 3 can also be applied for harmonic Ritz vectors. However, the computations become much more involved. Furthermore, extra ideas need to be developed for selecting the proper harmonic Ritz vectors. This is the subject of another paper.

\section{ACKNOWLEDGMENTS}

The authors thank Jan Brandts for suggestions that helped improve the presentation of this paper.

\section{REFERENCES}

[1] Ernest R. Davidson, The iterative calculation of a few of the lowest eigenvalues and corresponding eigenvectors of large real-symmetric matrices, J. Comput. Phys. 17 (1975), 87-94. MR 52:2168

[2] Zhongxiao Jia and G. W. Stewart, An analysis of the Rayleigh-Ritz method for approximating eigenspaces, Math. Comp. 70 (2001), no. 234, 637-647. MR 2001g:65040

[3] Shmuel Kaniel, Estimates for some computational techniques in linear algebra, Math. Comp. 20 (1966), 369-378. MR 38 \# 2934

[4] Andrew V. Knyazev, New estimates for Ritz vectors, Math. Comp. 66 (1997), no. 219, 985995. MR 97j:65090

[5] Beresford N. Parlett, The symmetric eigenvalue problem, Society for Industrial and Applied Mathematics (SIAM), Philadelphia, PA, 1998, Corrected reprint of the 1980 original. MR 99c:65072

[6] Y. Saad, On the rates of convergence of the Lanczos and the block-Lanczos methods, SIAM J. Numer. Anal. 17 (1980), no. 5, 687-706. MR 82g:65022

[7] Paul Smit, The approximation of an eigenvector by ritzvectors, Technical Report FEW 684, Center for Economic Research, University of Tilburg, Tilburg, The Netherlands, 1995.

[8] _ Numerical analysis of eigenvalue algorithms based on subspace iterations, Ph.D. thesis, Center for Economic Research, Tilburg University, Tilburg, The Netherlands, July 1997.

Department of Mathematics, Utrecht University, P.O. Box 80.010, NL-3508 TA Utrecht, The Netherlands

E-mail address: sleijpen@math.uu.nl

Department of Mathematics, Utrecht University, P.O. Box 80.010, NL-3508 TA Utrecht, The Netherlands

E-mail address: eshof@math.uu.nl

Center for Economic Research, Tilburg University, Tilburg, The Netherlands

Current address: IBM, Watsonweg 2, 1423 ND, Uithoorn, The Netherlands

E-mail address: p.smit@nl.ibm.com 